\title{
Northern Binaries in the Evrena Project
}

\author{
V. Bakış ${ }^{1}$, H. Hensberge ${ }^{2}$, M. Zejda ${ }^{3}$, P. de Cat ${ }^{2}$, F. Yılmaz ${ }^{4}$, \\ S. Bloemen ${ }^{5}$, P. Svoboda ${ }^{6}$ and O. Demircan ${ }^{4}$ \\ ${ }^{1}$ Akdeniz University, Space Sciences \& Technologies Dept., Campus, 07058, Antalya, Turkey \\ email: volkanbakis@akdeniz.edu.tr \\ ${ }^{2}$ Royal Observatory of Belgium, Brussels, Belgium \\ ${ }^{3}$ Masaryk University, Dept. of Theoretical Physics and Astrophysics, Brno, Czech Republic \\ ${ }^{4}$ Çanakkale Onsekiz Mart University, Physics Department and Ulupınar Observatory, \\ Terzioğlu Campus, TR-17020, Çanakkale, Turkey \\ ${ }^{5}$ Instituut voor Sterrenkunde, K.U.Leuwen, Celestijnenlaan 200D, B-3001, Leuwen, Belgium \\ ${ }^{6}$ Vypustky 5, Brno, Czech Republic
}

\begin{abstract}
In the framework of the EVRENA project, high-resolution spectra of northern eclipsing close binaries in stellar groups are obtained with the HERMES Echelle spectrograph at the Mercator telescope (Roque de los Muchachos Observatory). This contribution gives the first results on DV Camelopardalis.
\end{abstract}

Keywords. (stars:) binaries: eclipsing, (stars:) binaries (including multiple): close

\section{DV Camelopardalis}

DV Camelopardalis (HR 1719, HD 34233, HIP 24836, sp.type B5V) is a Cas-Tau OB association member with a membership probability of 99 per cent (de Zeeuw et al. 1999), and is known as a spectroscopic binary since early in the 20th century (Plaskett et al. 1921). The radial-velocity variability was confirmed by Petrie (1958), who mentioned an amplitude of $71 \mathrm{~km} \mathrm{~s}^{-1}$ from measurements at 12 epochs, and later by Fehrenbach et al. (1997). However, radial velocities were measured as if it was a single-lined binary, while our high-resolution spectroscopy presented here reveals three components (Fig. 1).

The Hipparcos satellite identified DV Cam as an eclipsing binary with an eclipse depth of roughly 0.2 magnitudes. The catalogue gives an orbital period of 1.5295 days. The light curve displays one eclipse. The scatter of the data within eclipse appears larger than expected from the estimated uncertainties in the photometry.

The Hipparcos photometric data set reveals 112 observations made at 21 epochs, of which 4 contain eclipse data. By 'epochs' we mean a series of observations with interruptions not exceeding significantly 0.1 day. The longest 'continuous' observation covers 1.95 days (24 data points) and contains 4 consecutive observations within eclipse, two at each side of mid-eclipse. This set alone already defines the duration of one of the eclipses as 0.15 to 0.2 days and suggests a mid-eclipse time at HJD 2448751.926 \pm 0.002 . The other 3 observations in eclipse are single observations (the second one, 20 minutes apart, was obviously rejected in the three cases), at the start or the end of a short series of observations. With only 4 epochs in eclipse, the question of whether the orbital period can be determined uniquely is relevant. Therefore, we made a search for possible periodicities in the interval 1.95 to 40 days starting with an analytical computation revealing all periods that (1) put the in-eclipse observations out of the phase intervals covered by series of observations at one epoch; and (2) group the in-eclipse observations in at most two phase intervals without any out-of-eclipse observations between them. 


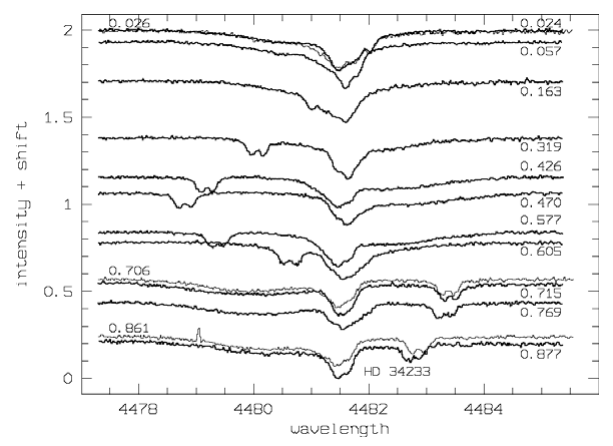

Figure 1. HERMES spectra of DV Cam. Thick lines: spectra taken 2011 Feb.17-27, thin lines: 2011 Jan. 9-11.

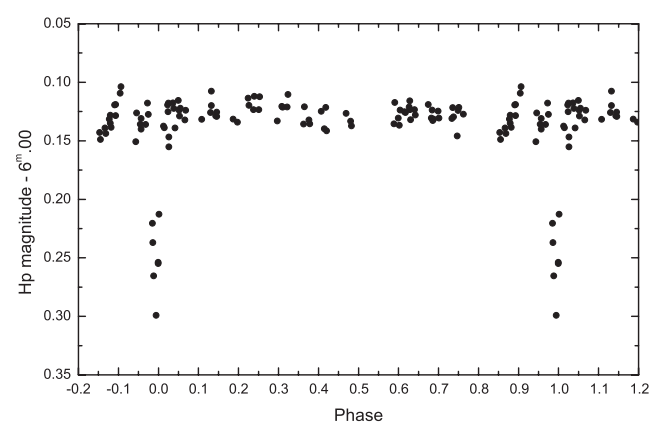

Figure 2. DV Cam light curve phased with ephemeris Pri HJD $=2448751.926+$ $6.67840 \times \mathrm{E}$.

The lower search limit is set by the longest series of observations, that contains only one eclipse. It must be shorter than the orbital period, as a nearly circular shorter period would imply two eclipses. Applying these criteria, 13 possible periodicities between 2.86 and 20.05 days were selected. Inspection of the phase diagrams identifies 6 candidate periods with adequately low scatter of the observations in eclipse(s): 3.0584 days (twice the Hipparcos period), 3.3392 days, and its multiples of $6.6784,10.0235,13.356$ and 20.047 days. We conclude that the Hipparcos data alone do not determine the orbital period in a unique way.

High-resolution spectroscopy of DV Cam was obtained in 2011 with the HERMES Echelle spectrograph (Raskin et al. 2011) attached at the 1.2-m Mercator telescope situated at the Roque de Los Muchachos Observatory. The spectra cover the wavelength range from close to the Balmer jump up to $9000 \AA$ with a resolution of 85000 . The highresolution spectra show clearly a contribution of three B-type stars (Fig. 1): a (quasi)stationary component, and a close-binary consisting of a sharp-lined and a broad-lined component with opposite Doppler shifts. The velocity range of the less massive sharplined stars sufficient to isolate its contribution at favourable orbital phases from those of the other components. The strength of the prominent lines of the (quasi)-stationary component suggests it is intermediate between the more massive component of the close binary and the cooler one.

The spectroscopy uniquely defines the true orbital period as $\mathrm{P}_{\text {orb }}=6.6784 \pm 0.0010$ days, with the uncertainty determined from the longer Hipparcos photometric time baseline (Fig. 2). The orbit is significantly eccentric, e $\sim 0.48$.

\section{Acknowledgements}

This work was supported by the Scientific Research Council of Turkey under project No. 109T449. Hermes is funded by the Fund for Scientific Research of Flanders (FWO) grant G.0472.04, the Research Council of K. U. Leuven grant GST-B4443, the Fonds National de la Recherche Scientifique contracts IISN4.4506.05 and FRFC 2.4533.09, and the Royal Observatory of Belgium Lotto(2004) fund.

\section{References}

Fehrenbach, C., Duflot, M., Mannone, C., Burnage, R., \& Genty, V. 1997, A\&AS, 124, 255

Petrie, R. M. 1958, MNRAS, 118, 80

Plaskett, J. S., Harper, W. E., Young, R. K., \& Plaskett, H. H. 1921, Publ. DAO, 1, 287

Raskin, G., Van Winckel, H., Hensberge, H., et al., 2011, A\&\&A, 526, 69

de Zeeuw, P. T., Hoogerwerf, R., de Bruijne, J. H. J. et al., 1921, AJ, 117, 354 